\title{
Discrete Element Method Study on the Evolution of Contact Force inside Railway Ballast under Tamping Operation
}

\author{
Taoyong Zhou ${ }^{1, \text { a }}$, Bin $\mathrm{Hu}^{2}$, Junfeng Sun ${ }^{1}$ and Bo Yan ${ }^{1}$ \\ ${ }^{1}$ Faculty of Mechanical and Electrical Engineering, Kunming University of Science and Technology, \\ Kunming 650500, China \\ ${ }^{2}$ China Railway Construction High-tech Equipment Co., Ltd., Kunming 650215, China \\ akmzty@163.com
}

Keywords: Contact Force, Railway Ballast, Tamping, EDEM.

\begin{abstract}
The tamping operation is employed to restore the geometry of railway track distorted by train traffics. In order to study the micro mechanical properties of railway ballast under tamping operation, the analysis model of railway ballast under tamping operation is created using the discrete element method, numerical simulations are performed to study the evolution of contact force inside railway ballast under tamping operation. The obtained results are showed that the contact force inside railway ballast is enhanced by tamping operation, so as to improve the carrying capacity and stability of railway track.
\end{abstract}

\section{Introduction}

Railway ballast is the railway track foundation that is composed of ballast gravel. With the increasingly busy of rail transport, the railway line will be inevitably deformed or damaged. In order to restore the geometry of railway track, the maintenance work of the railway line need strengthen to keep the equipment of railway line in good condition. When performing railway track maintenance, tamping is usually adopted to adjust the longitudinal profile, the cross level, and the alignment of the track, which is the geometrical parameter that most influences the vehicles and the track dynamics in the vertical direction. So the tamping operation is an extremely important work in the maintenance of the railway line. As the ballast gravel of railway is granular media of different shapes and sizes, during tamping process, the mechanical properties of railway ballast is difficult to measure and describe, the research of the micro mechanics of railway ballast is difficulties, the selection of tamping parameters is usually dependent on field trials and practical experience, for lack of theoretical basis [1,2].

In recent years, the rapid development of discrete element method offers a new means of studying the response characteristics of railway ballast during tamping process [3]. The discrete element method was developed based on the principle of molecular dynamics by the American scholar P. A. Cundall in 1971, which is used to study the discrete particle materials. The basic idea of discrete element method is that arbitrary discontinuities are divided into a set of rigid elements, make each rigid element satisfy the equations of motion, use time step iteration method for solving the equations of motion of rigid elements, and then obtain the overall movement patterns of arbitrary discontinuities [4-6]. After more than forty years, continuous in-depth study, the discrete element method is more widely used in research areas involving solid particles [7-10].

It is regarded as a new attempt that the use of discrete element method for creating discrete element model of railway ballast and simulating the evolution of contact force inside railway ballast under tamping operation.

\section{Discrete element analysis model}

Discrete element analysis model of railway ballast during tamping process is composed of particle model of ballast gravel, geometry model of steel rails, geometry model of sleepers, geometry model of tamping tines, particle factories of railway ballast and so on [11]. This paper 
tries to create the analysis model of railway track using the discrete element method in the discrete element analysis software EDEM.

Particle model. At present the basic particle shape model is sphere in the three-dimensional discrete element analysis software, however, the shape of ballast gravel is irregular, the size of ballast gravel is also inconsistent, which brings some difficulties to simulation analysis. In the discrete element analysis software EDEM, overlapping spherical surfaces of different sizes is used to form complex clumps in order to simulate particles of irregular shape, this paper creates two typical particle model to simulate the real ballast gravel by mean of overlapping spherical surfaces of different sizes, as shown in Fig. 1.
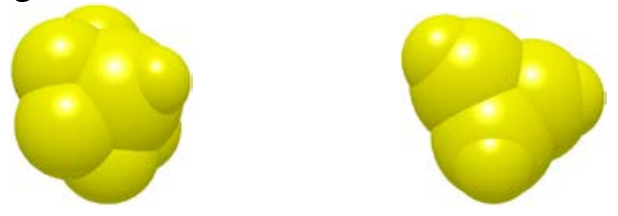

Fig. 1 Particle model of ballast gravel

Geometry model. Geometry model can be defined in EDEM or imported from a CAD package. The geometry model of steel rails, sleepers and tamping tines are created in Solid Edge, and then imported into EDEM. In addition to the Poisson's ratio, shear modulus and density of the materials properties of steel rails, sleepers and tamping tines, the dynamic properties of tamping tines should be defined in EDEM. This paper defines the dynamic properties of tamping tines through the reference to the actual industrial tamping operation process, the first phase is penetration of tamping tines into the ballast, the second phase is squeezing of ballast gravel under the sleeper, the third phase is keeping the squeezing, the fourth phase is releasing the squeezing, and the fifth phase is lifting tamping tines from the ballast, as well as the tamping tines always maintain the vibration throughout the tamping operation process.

Particle factories. Particle factories are used to define where, when and how particles appear in a simulation. Any virtual surface or volume can be turned into a particle factory. For simulating the railway ballast bed, a virtual box is created to be turned into a particle factory, and some associated parameters are defined in this paper.

The discrete element analysis model of railway track during tamping process has been created by creating the particle of ballast gravel, the geometry model of steel rails, sleepers and tamping tines, and particle factories of railway ballast, as shown in Fig. 2.

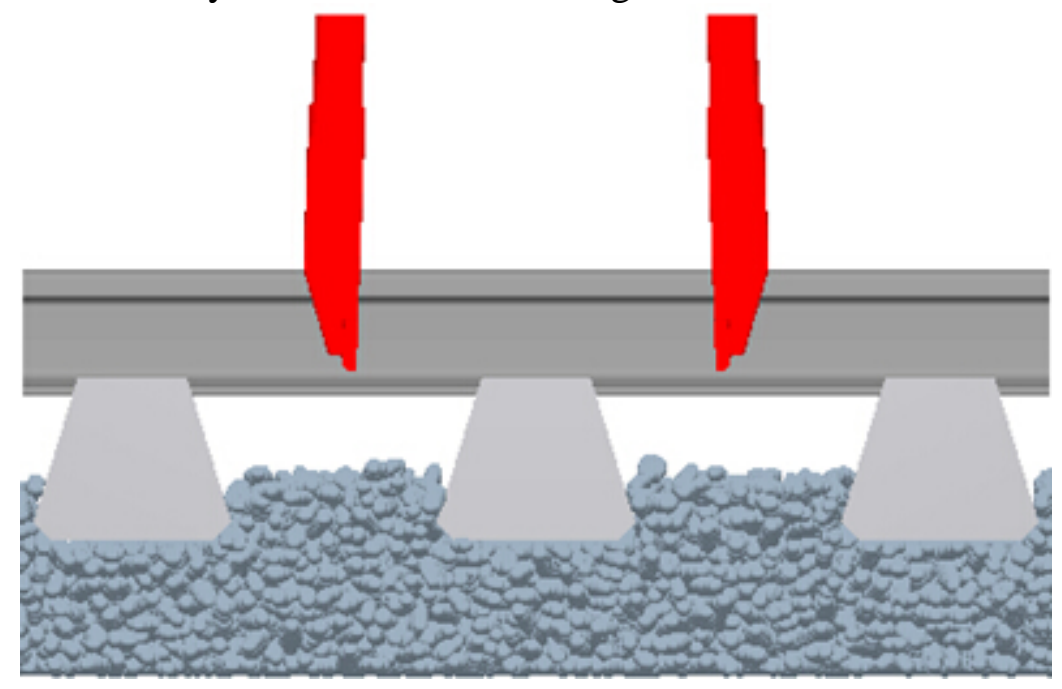

Fig. 2 Analysis model of tamping operations

\section{Simulation analysis}

In order to study the micro mechanical behavior of railway ballast under tamping operation, this paper presents the evolution of contact force inside railway ballast under tamping operation. 
In granular materials, force is transmitted from one particle to the next via their contacts. In order to visualize describe the propagation of force, the individual contact force between particles is identified by line. The thickness of line represents the magnitude of contact force; the direction of arrow represents the direction of contact force [12,13].

In order to study the evolution of contact force inside railway ballast during tamping process, the contact force that develop inside the railway ballast at three different stages of the simulation are plotted as lines with thickness proportional to force magnitude, as shown in Fig. 3.

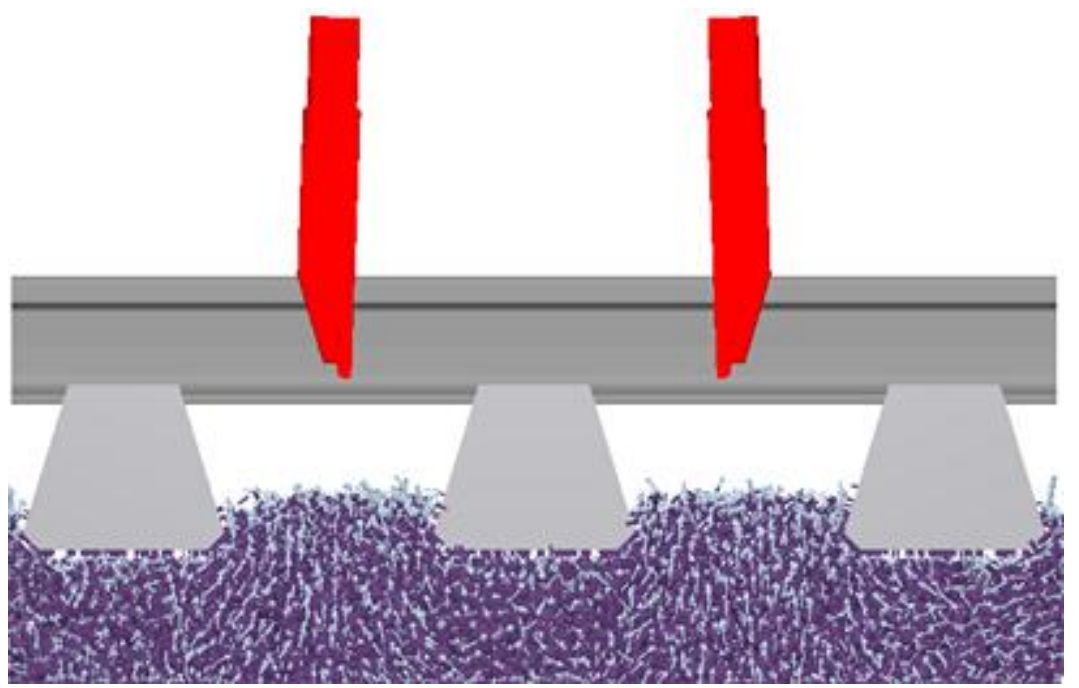

(a)

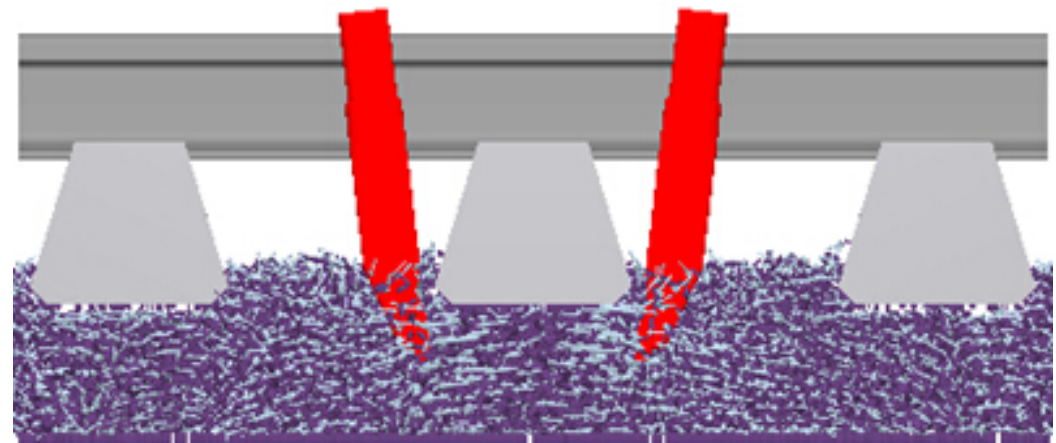

(b)

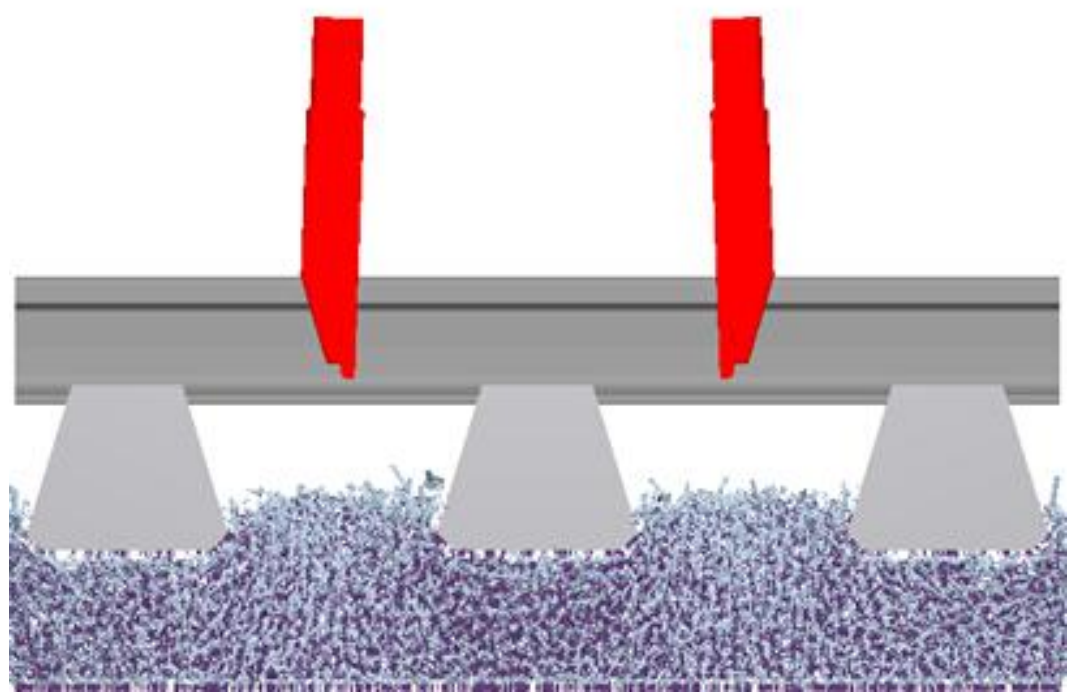

(c)

Fig. 3 Contact force. (a) Before tamping (b) During tamping and (c) After tamping 
By comparing the thickness of lines which represents the magnitude of the contact force inside railway ballast at three different stages of the simulation, it is found that the lines after tamping are thicker than the lines before tamping, which means that the contact force inside railway ballast is enhanced by tamping, so as to improve the carrying capacity and stability of railway track.

\section{Summary}

The discrete element analysis methods has been used to simulate analysis the micro mechanical behavior of railway ballast under tamping operation, according to the discrete characteristics of railway ballast.

The simulation analysis is carried out to study the evolution of contact force inside railway ballast under tamping operation. The lines after tamping are thicker than the lines before tamping, which mean that the contact force inside railway ballast is enhanced by tamping, so as to improve the carrying capacity and stability of railway track.

The obtained results are consistent with those of other similar studies, which mean that the discrete element method is an effective method to study the micro mechanical behavior of railway ballast under tamping operation. Future work will be further study the mechanical behavior of railway ballast during tamping process under different tamping parameters.

\section{Acknowledgements}

This work was financially supported by the Yunnan Province Education Department Scientific Research Foundation (2014Y056).

\section{References}

[1] T.Y. Zhou, B. Hu and J.F. Sun: Journal of Applied Sciences Vol.13 (2013), p.2072.

[2] G. R. McDowell, W. L. Lim, A. C. Collop, R. Armitage and N. H. Thom: Transport Vol. 158 (2005), p. 89.

[3] G. Saussine, E. Azema, R. Peales and F. Radjai: Powders and Grains Vol. 1145 (2009), p. 469.

[4] G. M. Hu: Analysis and simulation of granular system by discrete element method using EDEM. (Wuhan University of Technology Press, Wuhan 2010).

[5] P. A. Cundall and O. D. L. Strack: Geotechnique Vol. 29 (1979), p. 47.

[6] D. O. Potyondy and P. A. Cundal: International Journal of Rock Mechanics and Mining Sciences Vol. 41 (2004), p. 1329.

[7] W. L. Lim and G. R. McDowell: Granular Matter Vol. 7 (2005), p. 19.

[8] M. Lu and G. R. McDowell: Geotechnique Vol. 60 (2010), p. 459.

[9] G. Q. Jing, K. Feng, L. Gao and J. Wang: Journal of Southwest Jiaotong University Vol. 47 (2012), p. 187.

[10] Q. D. Sun, B. Indraratna and S. Nimbalkar: Geotechnique Vol. 64 (2014), p. 746.

[11] T. Y. Zhou, B. Hu, J. F. Sun and Z. T. Liu: Open Electrical and Electronic Engineering Journal Vol. 7 (2013), p. 103.

[12] J. F. Peters, M. Muthuswamy, J. Wibowo and A. Tordesillas: Physical Review E-Statistical, Nonlinear, and Soft Matter Physics Vol. 72 (2005), p. 1.

[13] S. Lobo-Guerrero and L. E. Vallejo: Granular Matter Vol. 8 (2006), p. 195. 